\title{
Effects of surface disorder and temperature on atomic friction
}

\author{
O. Y. Fajardo* and J. J. Mazo \\ Departamento de Física de la Materia Condensada and Instituto de Ciencia de Materiales de Aragón, \\ CSIC-Universidad de Zaragoza, 50009 Zaragoza, Spain
}

(Received 26 February 2010; revised manuscript received 5 July 2010; published 26 July 2010)

\begin{abstract}
We have investigated the effect of surface disorder on atomic friction in the stick-slip dynamical regime of a one-dimensional Prandtl-Tomlinson model. We find that disorder in the substrate potential can modify the mean slip length and then it changes the friction force of the system. In particular, the effect of the disorder is more important at low temperatures and close to dynamical transition points or at high loads. We show results for different values of temperature, amplitude of the corrugation potential, dragging velocity, and damping. Special care is put in the comparison between the results of the regular and the disordered cases at many different temperatures and in the understanding of the combined effect of disorder and temperature in the dynamics of the system. To finish, we present some results on the robustness of the results against a change in the effective damping of the system.
\end{abstract}

DOI: 10.1103/PhysRevB.82.035435

PACS number(s): 68.35.Af, 81.40.Pq, 68.37.Ps, 46.55.+d

\section{INTRODUCTION}

Nanotribology is the science that studies adhesion, friction, wear, and lubrication phenomena. ${ }^{1-3}$ The understanding of friction at the nanoscale is a current important problem from both a fundamental and a technological perspective. ${ }^{4,5}$ On the one hand the comprehension of friction at the nanoscale is fundamental for the manipulation of nanoparticles and the miniaturization of moving devices as nanoelectromechanical systems, for instance. On the other hand friction is a complex nonlinear phenomenon of fundamental interest in many scientific areas. ${ }^{6,7}$ With the development of new experimental techniques such as the atomic force microscopy and its modified version, the force friction microscopy, and the surface force apparatus, experimental and theoretical studies of friction at the atomic scale have received a significant attention. Furthermore, as the interaction between two surfaces involves complex interactions among many asperities, the atomic force microscopy is an exceptional tool to better understand friction at nanoscale level since it can be described essentially as a single asperity dragged along a surface. . $^{4}$

Most theoretical efforts to describe the force friction microscopy experiments have focused on the behavior of defect free and perfect periodic surfaces with or without the inclusion of thermal effects. ${ }^{9-12}$ However, the study of the effect of substrate disorder or defects on atomic friction is particularly important since atomically flat surfaces represent ideal models and disorder and defects of different kind are always present in nature. Recent results in an one-dimensional model indicate that the presence of surface defects can modify the temperature dependence of friction at atomic scale. ${ }^{13}$ Braiman et al. ${ }^{14}$ have also presented results which indicate that quenched disorder and thermal noise can decrease friction. Hölscher et $a l .{ }^{15}$ analyzed the load dependence of atomic friction at atomic-scale surface steps.

The aim of this paper is to study the effect of surface disorder on atomic friction and its interplay with thermal effects. We will focus in the stick-slip region of the friction force versus dragging velocity curve. In particular, we present results for the mean friction force and slip length for a range of values of the corrugation potential amplitude at different driving velocities and damping. By surface disorder we mean small deviations from the perfect lattice model. Such deviations are mainly due to the uncertainty in the strength of the effective interaction between the tip and the surface. We present numerical results that indicate that such effects may strongly modify the frictional behavior at atomic scale. This change in the friction force results from a change in the mean slip length. In addition, we find important changes in the friction force due to small changes in the damping in the small damping case.

\section{MODEL}

We use a generalized Prandtl-Tomlinson model which includes the thermal effects ${ }^{12}$

$$
\begin{gathered}
M \frac{d^{2} x}{d t^{2}}+M \gamma \frac{d x}{d t}+\frac{\partial U(R, x)}{\partial x}=\xi(t), \\
U(R, x)=\frac{k}{2}[R(t)-x]^{2}+V(x) .
\end{gathered}
$$

In this picture the tip is modeled as a single particle dragged over a one-dimensional substrate potential. Here $U(R, x)$ is the tip effective potential, which includes the elastic coupling effect of the tip with a support which moves at a constant velocity $v_{s}\left[R(t)=R_{0}+v_{s} t\right]$, and the tip-surface interaction $V(x) . M$ and $x$ are the effective mass and the lateral position of the tip and $k$ is a spring constant. $\xi(t)$ is the random noise term which satisfies the fluctuation-dissipation relation $\left\langle\xi(t) \xi\left(t^{\prime}\right)\right\rangle=2 M \gamma k_{\mathrm{B}} T \delta\left(t-t^{\prime}\right)$ with $\gamma$ the microscopic friction coefficient and $k_{\mathrm{B}}$ the Boltzmann constant.

We model surface disorder including a second harmonic term in the standard tip-surface interaction potential

$$
V(x)=-U_{o}\left[1.0+\epsilon \sin \left(\frac{2 \pi x}{b}\right)\right] \cos \left(\frac{2 \pi x}{a}\right) .
$$

Here $\eta=\frac{a}{b}$ is the commensurability factor, $\epsilon$ measures the importance of the second harmonic, and $a$ and $U_{o}$ are the 


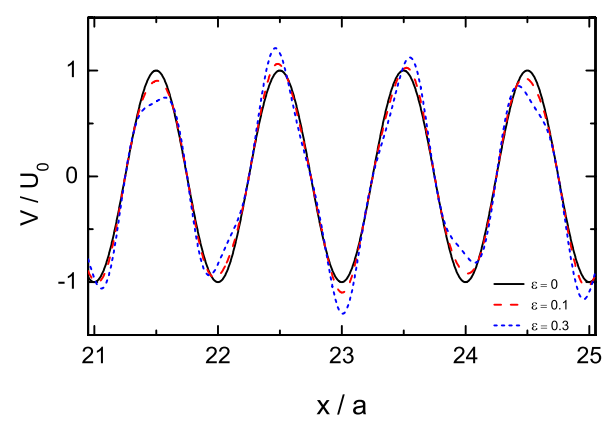

FIG. 1. (Color online) Tip-surface potential for $\eta=\frac{\sqrt{5}+1.0}{2.0}$ and $\epsilon=0$ (perfectly sinusoidal lattice), $\epsilon=0.1$ (weak disorder, red dashed line), and $\epsilon=0.3$ (strong disorder, blue dotted line).

lattice spacing and the amplitude of the regular $(\epsilon=0)$ surface potential, respectively. In theory, this amplitude can be changed by varying the normal load ${ }^{10,16}$ although it is worth noting that a change in the normal load is probably changing other parameters of the system in a nontrivial way. The effect of this disorder is a small change in the potential shape (see Fig. 1). It introduces a distribution of barrier heights and slightly moves the positions of the potential maxima and minima.

We are mainly interested in studying the mean value $\langle F\rangle$ of the instantaneous friction force $F(t)=k[R(t)-x(t)]$ as a function of the driving velocity $v_{s}$, temperature, interaction strength $U_{o}$, and the other parameters of the model. Nondimensional equations are obtained if we measure energy in units of $U_{0}$, space in units of the lattice spacing $(\tilde{x}=2 \pi x / a)$ and time in units of the natural frequency for oscillations of the tip in the surface potential $\left(\tau=\omega_{p} t\right.$ with $\omega_{p}$ $\left.=2 \pi \sqrt{U_{o} / M a^{2}}\right)$. Then $\tilde{\gamma}=\gamma / \omega_{p}, \tilde{k}=1 / \Theta=k a^{2} /\left(4 \pi^{2} U_{o}\right)$, and $\widetilde{v_{s}}=v_{s} \sqrt{M / U_{o}}$ are the scaled damping, spring constant, and velocity, respectively.

We have numerically integrated Eq. (1) for different values of the system parameters. Following the work by Tshiprut et al., ${ }^{13}$ in our simulations we have used $M=5.0$ $\times 10^{-11} \mathrm{Kg}, a=0.45 \mathrm{~nm}, k=1.5 \mathrm{~N} / \mathrm{m}, \gamma=10^{5} \mathrm{~s}^{-1}$ (except in Sec. $\mathrm{V}$ where we change the damping), and $U_{0}$ in the $0.2-1.2 \mathrm{eV}$ range (then $\tilde{\gamma}$ goes from 0.3 to 0.1 and $\Theta$ from 4 to 25$)$. For $v_{s}$ we have normally used $10 \mathrm{~nm} / \mathrm{s} .{ }^{17}$ Regarding the surface disorder potential we have used $\eta=(\sqrt{5}$ $+1.0) / 2.0$ and $\epsilon=0,0.1$, or 0.3 .

\section{SURFACE DISORDER WITHOUT TEMPERATURE}

In this section we present numerical simulations of the effect of surface disorder as defined in Eq. (2) on the physical properties of the system, mainly the average friction force $\langle F\rangle$, at different parameter values. Before doing so, in order to get a better insight on the problem we will briefly describe some of the main properties of the system for a perfect lattice $(\epsilon=0) .4,6,8-13$ For this case, the main results are shown in Fig. 2(a) where we present the friction force as a function of the corrugation potential amplitude $U_{o}$ at small velocity $\left(v_{s}\right.$ $=10 \mathrm{~nm} / \mathrm{s}$ ). The inset shows a typical friction force versus driving velocity curve (computed at $U_{0}=0.27 \mathrm{eV}$ ). Here,
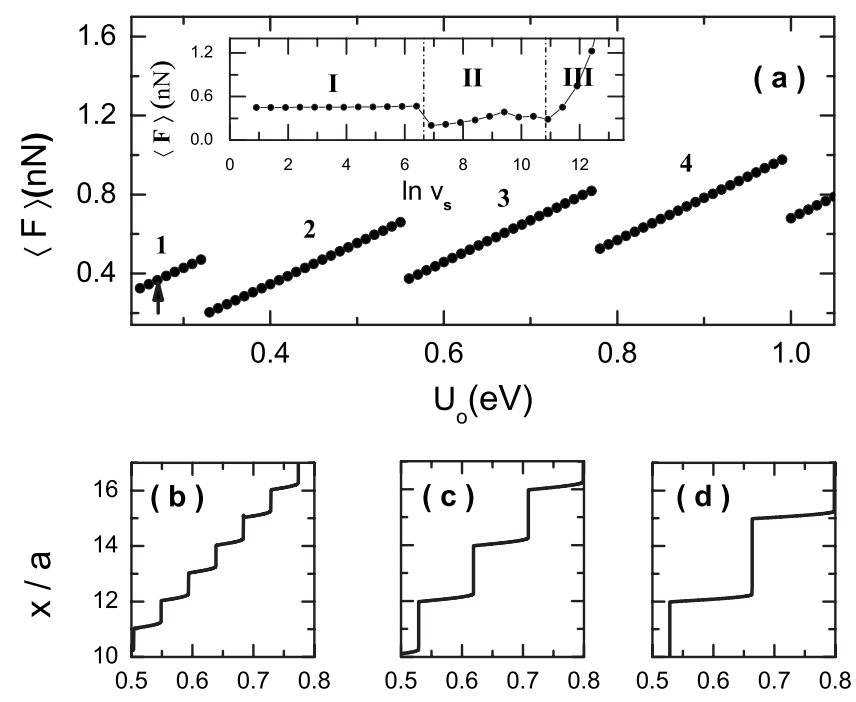

$t(s)$

FIG. 2. (a) Friction versus corrugation potential amplitude $U_{o}$ $\left(v_{s}=10 \mathrm{~nm} / \mathrm{s}\right)$ and (inset) friction versus driving velocity at $U_{o}$ $=0.27 \mathrm{eV}$, see arrow. Figures (b), (c), and (d) show the time dynamics of the tip for different values of $U_{o}$ corresponding to regions 1,2 , and 3 in (a). In all the cases $T=0 \mathrm{~K}$.

and in the figures below, $\ln v_{s}$ refers to a velocity measured in $\mathrm{nm} / \mathrm{s}$. In all the cases $\gamma=10^{5} \mathrm{~s}^{-1}$.

In the curve of the friction force versus the driving velocity [inset of Fig. 2(a)], we distinguish between three different dynamical regions. At low velocities a first region (marked as I) is found which corresponds to the well-known stick-slip behavior with one-lattice constant slips. ${ }^{18}$ The second region (region II) is also characterized by the stick-slip behavior but with larger slip events. This second region appears usually for intermediate values of velocity and it is not found at high-enough damping. Finally, we find region III which corresponds to higher velocities where the tip slides smoothly and the stick-slip behavior disappears.

We will mainly focus on the dynamics of the system in the stick-slip regions. There, the dynamics is governed by the length of the slip events. ${ }^{9,10,19}$ Figure 2(a) shows the friction force as a function of the potential amplitude, $U_{0}$. This curve presents a series of marked discontinuities which correspond to a change in the length of the slip events. The discontinuities mark transitions between different dynamical states. The regions numbered as 1, 2, 3, and 4 in the figure correspond, respectively, to one, two, three, and four-lattice constant jumps. The range of values of the parameter $\Theta$ in each region are: $1.0<\Theta_{1} \leq 6.66 \leq \Theta_{2} \leq 11.45 \leq \Theta_{3} \leq 16.03 \leq \Theta_{4}$ $\leq 20.61$. For values of $\Theta<\Theta_{1}$, the tip slides smoothly, stickslip events do not appear. Figures 2(b)-2(d) show the time dynamics of the tip for regions 1,2 , and 3 .

It is important to mention here that a change in $U_{o}$ also changes the nondimensional damping and velocity of the system since $\tilde{\gamma}=\gamma / \omega_{p}$ with $\omega_{p}=2 \pi \sqrt{U_{o} / M a^{2}}$ and $\widetilde{v_{s}}$ $=v_{s} \sqrt{M / U_{o}}$. Thus a high $U_{o}$ implies a smaller $\tilde{\gamma}$ and it allows longer slip events. Following previous studies, ${ }^{13,17}$ we consider $U_{o}, \gamma$, and $v_{s}$ as independent parameters which can be somehow experimentally controlled or measured. Moreover, 

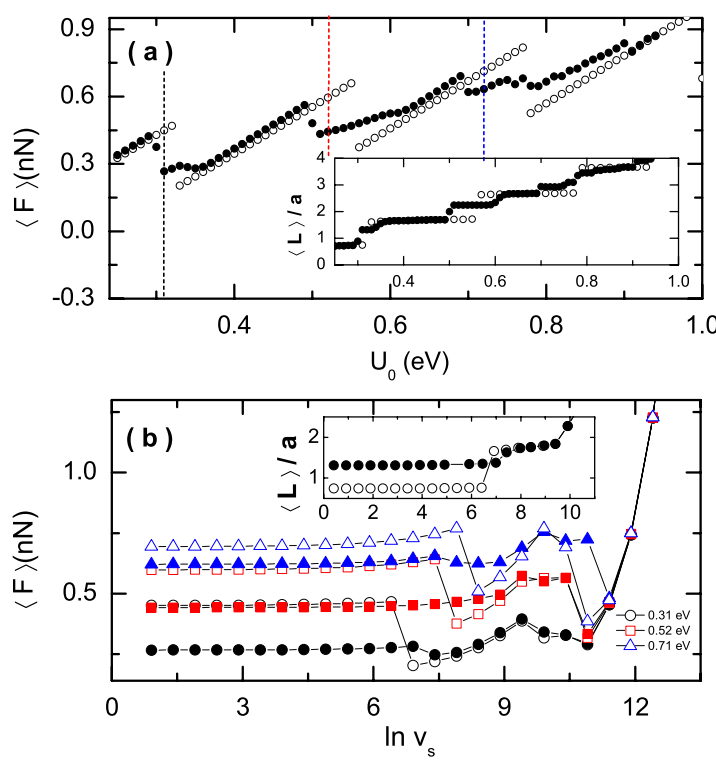

FIG. 3. (Color online) Surface disorder effect in the stick-slip dynamics of the system at $T=0 \mathrm{~K}$. Open symbols are for the regular $(\epsilon=0)$ potential and solid ones for the surface disorder case $(\epsilon=0.1)$. (a) Friction force and mean slip length versus potential amplitude in the low velocity regime. The inset shows the average slip length at the different $U_{0}$. (b) Friction force as a function of the velocity $v_{s}$ for $U_{o}=0.31 \mathrm{eV}$ (circles), $0.52 \mathrm{eV}$ (squares), and 0.71 $\mathrm{eV}$ (triangles). The inset shows the mean slip length for $U_{0}$ $=0.31 \mathrm{eV}$.

we have numerically checked that the transitions observed in the figure are also observed in simulations at constant $\tilde{\gamma}$ and $\widetilde{v_{s}}$ and thus can be ascribed to modifications in the potential profile.

Now we are going to present result on how the inclusion of surface disorder affects the friction force and the dynamics of the tip. We will see that, in some cases, surface disorder strongly modifies the response of the system. As expected, the main effect of disorder is to smooth the friction versus corrugation potential curves. However, a small amount of disorder also affects the characteristic curves importantly at some parameter values, in particular, close to the transition points, see Fig. 3(a). In these regions the presence of disorder changes the value of the mean length of the slip events (see inset) which has important consequences in the average friction force result. The figure also shows that the effect of disorder is more important at large values of $U_{0}$. There, the dimensionless damping is smaller and the dynamics is more sensitive to small changes in the substrate potential.

Figure 3(b) shows a series of friction force versus driving velocity curves for various values of $U_{0}(0.31,0.52$, and 0.71 $\mathrm{eV}$ ) and it compares the regular (open symbols) and the disordered (solid symbols) cases. Such $U_{0}$ values have been chosen close to the dynamical transition points. In the low velocity part of the curve (region I) the friction force value has been importantly modified by the presence of surface disorder. At higher velocity values the system switches into region II and this difference disappears. This region is characterized by larger slip events which correspond to a smaller
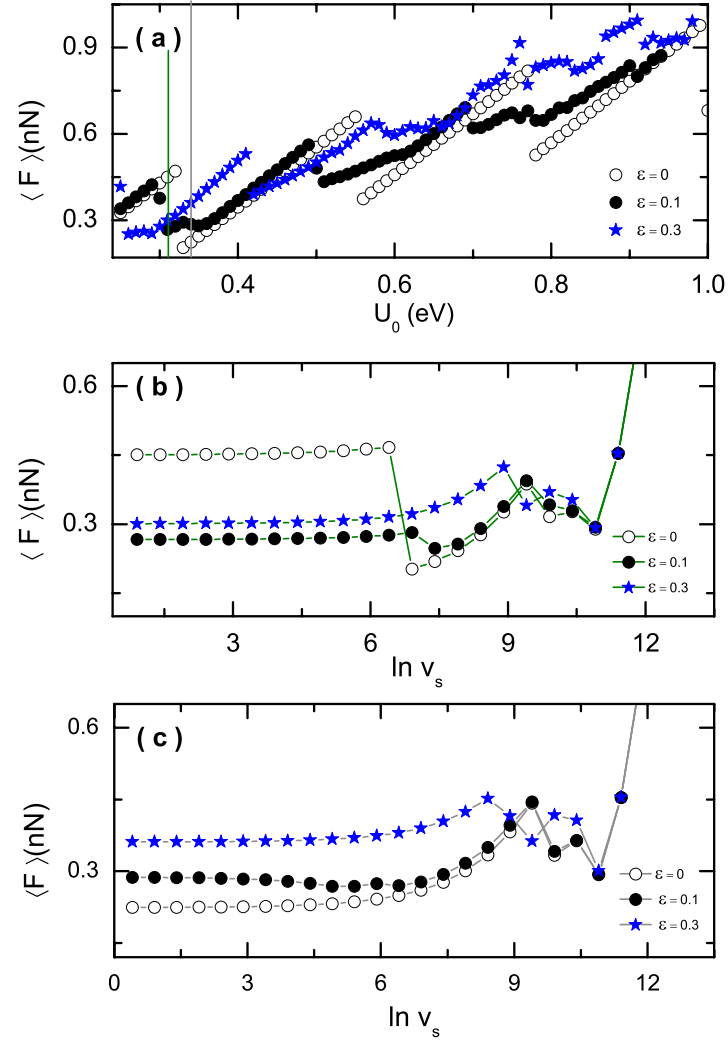

FIG. 4. (Color online) Friction force versus potential at $v_{s}$ $=10 \mathrm{~nm} / \mathrm{s}$, figure (a); and friction force versus velocity at $U_{0}$ $=0.31 \mathrm{ev}$, figure (b), and at $U_{0}=0.34 \mathrm{eV}$, figure (c), for $\epsilon=0,0.1$, and 0.3 .

mean friction force. The abrupt transition observed in the regular case is smoothed and anticipated by the presence of disorder. At high velocities the stick-slip phenomenon disappears and there is no difference between the regular and the disordered situations. Again, we can look at the mean slip length curve [inset of Fig. 3(b)] to understand the previous curve in terms of this variable. The difference between the regular and the disordered cases is caused by the modification of the slip length of the tip due to the inclusion of disorder and depend on the specific values of $U_{o}$ and $\gamma$.

Figure 4 shows the effect of a larger disorder in the characteristic curves of the system. As said above, close to the transition points the inclusion of a small quantity of disorder can produce a dramatic change in the response of the system. We also see that for $\epsilon=0.3$ although the perturbation of the regular profile is just a bit larger than that for $\epsilon=0.1$ (see Fig. $1)$, the effect on the friction force is much stronger. At this value of disorder important overlaps between areas corresponding to different slip lengths are observed. Values of disorder beyond this value of $\epsilon=0.3$ produce larger effects.

\section{SURFACE DISORDER WITH TEMPERATURE}

Now we will study the combined effect of surface disorder and temperature in the dynamics of the system. There are many theoretical and experimental evidences showing the important role played by temperature in the friction 

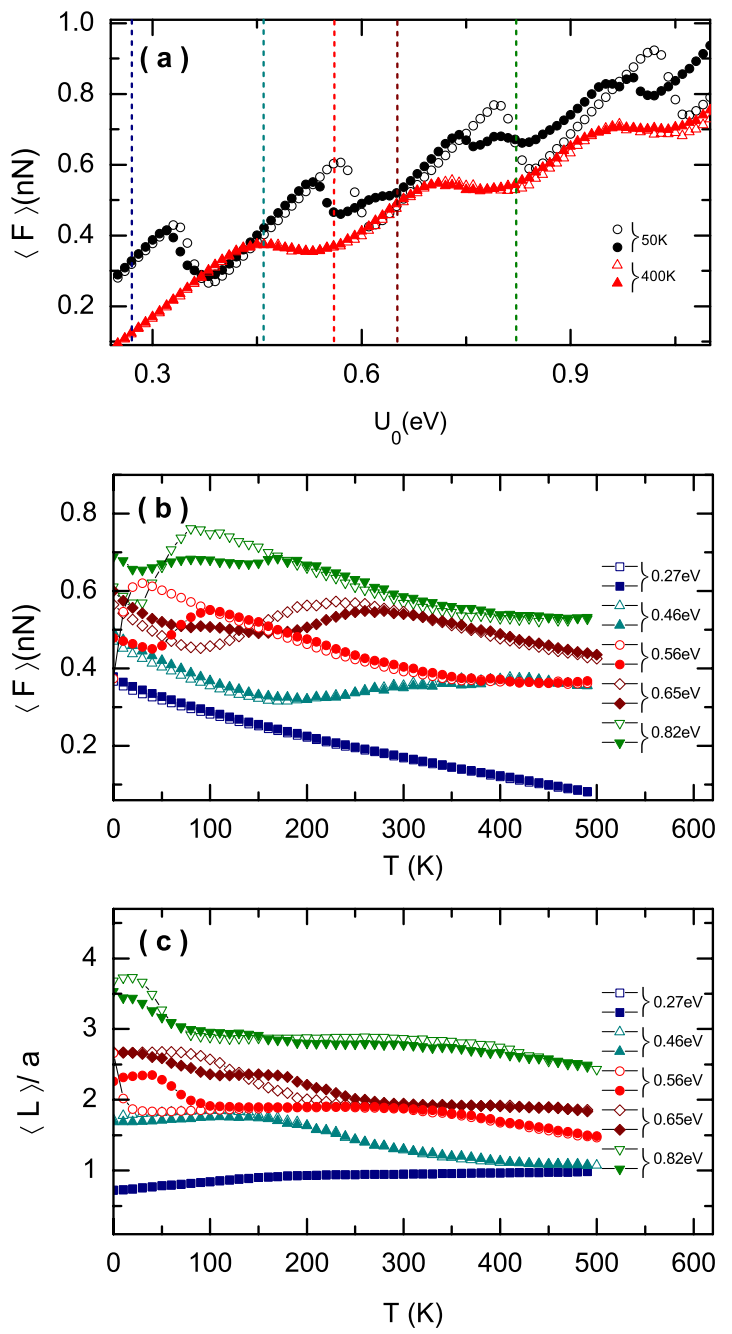

FIG. 5. (Color online) Comparison of the $\epsilon=0$ (open circles) and the $\epsilon=0.1$ (solid circles) curves at different values of $T$ and $U_{0}$. (a) $\langle F\rangle$ versus $U_{0}$ at 50 and $400 \mathrm{~K}$. (b) $\langle F\rangle$ versus $T$ and (c) $\langle L\rangle$ versus $T$ at $U_{0}=0.27,0.46,0.56,0.65$, and $0.82 \mathrm{eV}$.

phenomena. ${ }^{11-13,20-23}$ The main temperature effect is the reduction in the friction force in the stick-slip region of the friction versus driving velocity curves. This reduction has been observed at high temperatures and is explained by the existence of thermally activated jumps of the tip. ${ }^{11,12}$ However, it has been recently observed that at low temperatures thermal fluctuation can increase the friction force which reaches a maximum and then decreases. ${ }^{13,23}$ In addition to thermal activation, the presence of temperature can also reduce the mean length of the slip events. This reduction explain the increase in the friction force which dominates the behavior of the system at low temperatures. ${ }^{13}$ Such effects are clearly seen in Figs. 5(b) and 5(c), where results for the regular potential (open symbols) are also shown.

Due to the important role played by temperature it is natural to consider now the combined effect of thermal fluctuations and surface disorder in the response of the system. Figures 5 and 6 summarize the main results. In Fig. 5(a) we compare the friction versus the potential amplitude curve of the regular $(\epsilon=0)$ and the irregular case $(\epsilon=0.1)$ at 50 and $400 \mathrm{~K}$ [for $T=0 \mathrm{~K}$ see Fig. 3(a)]. The more important dif-
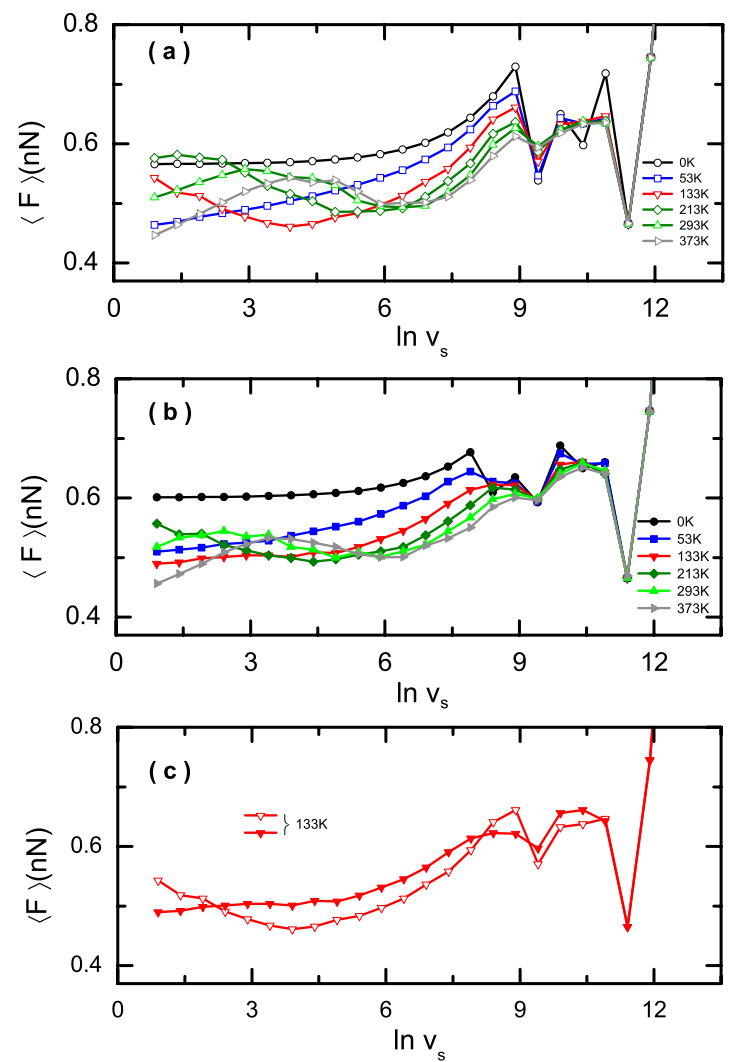

FIG. 6. (Color online) Force versus velocity curves for (a) $\epsilon$ $=0$ and (b) $\epsilon=0.1$ at different values of $T$ and for $U_{o}=0.65 \mathrm{eV}$. Figure 6(c) compares the result for the regular and for disordered case at $T=133 \mathrm{~K}$.

ferences between both curves are observed at small temperature values and close to the transition points or at high values of the potential amplitude. As it can be seen in the figures the differences disappear at high temperatures. This effect is studied in Figs. 5(b) and 5(c), where we have computed the friction force and the mean length of the slip events as a function of the system temperature for five different values of $U_{o}$ corresponding to the five different dynamical states marked in Fig. 5(a).

It is observed that the inclusion of disorder does not modify the two main competing thermal effects already reported in the literature, see Figs. 5(b) and 5(c): monotonic decreasing of friction due to thermal activation without modification of the mean slip length, dominant at low values of $U_{0}$ or high temperatures, and increasing of friction due to reduction of the slip length, observed at low temperatures and high $U_{0}$ values. This reduction can be understood using the stick-slip picture of the dynamics of the tip where the potential profile of the tip, which also depends on the relative position between the tip and the support, changes continuously developing new minima during the stick part of the cycle which allows for long jumps. Thermal noise shortens the sticking time by inducing thermally activated slips. Thus, the potential profile contains a smaller number of accessible minima and the mean length of the slips also shortens.

As expected, regarding the inclusion of disorder it is observed that strong-enough thermal fluctuations are able to 
screen any effect induced by disorder. We have also studied the combined effect of disorder and temperature on the friction force versus support velocity curves. Figure 6 shows examples of the modification of the friction force versus velocity curves of the system due to the combined effect of disorder and temperature. We can distinguish the three regions shown in Fig. 2(a) and the two main thermal effects described above. Figure 6 also shows, in some cases, a reduction in the friction force with the velocity in the lowvelocity region of the system characteristic curve. A similar result has been recently reported by Barel and Urbakh. ${ }^{24}$

\section{DAMPING EFFECT}

Thermal effects have been included by assuming the existence of a heat bath at a given temperature coupled to the system and satisfying the fluctuation-dissipation relation. Such dissipation is controlled by the damping parameter, which can be seen as a measure of the intensity of the coupling of the system to the bath, which models the effect of the many other degrees of freedom present in the system. Temperature is a well-controlled parameter. However, to determine the correct value of the damping for the tip is a much more complex and difficult question. Thus, it is important to study the dynamics of the system for different values of the damping in order to check the robustness of the results against changes in this parameter. The effect of damping has been studied in the context of the robustness of the functional form of the velocity dependence of the friction force ${ }^{25}$ and to study the effect of tip flexibility on the stick-slip dynamics. ${ }^{26}$

Concerning damping effects, it is usually defined a critical damping value above which the tip oscillations disappear (overdamped dynamics). In this case all the deterministic slips have a length close to one lattice constant. Some experiments indicate that damping is below this critical value, which corresponds to a more complex and dynamically richer situation. ${ }^{27}$

We have investigated first in the regular case the effect of the damping parameter on the friction force for values of $\tilde{\gamma}$ crossing the critical value. Then we analyze how this behavior is affected when surface disorder effects are included. Our first finding, Fig. 7(a) shows a series of steps in the friction force versus damping curve. Close to the discontinuities a small change in the damping can produce a large change in the friction force. Every step in this figure corresponds to a slip close to one or two lattice sites, respectively (see also inset). In the overdamped limit only site 1 is reached. At smaller values of the damping the tip can reach site 2. If damping is reduced again the tip can move though 1 to 2 and then oscillates back to 1 where is trapped. At small damping the tip oscillates back and forth between both minima before reaching an equilibrium value. Then, by decreasing the value of the damping more transitions are observed. Figures 7(b) and 7(c) show the time evolution of the position and force, respectively, of the tip for three close values of the damping, two of them at both sides of a transition point. For other values of the parameters (larger $U_{0}$ ), more complex situations are found. In these cases the dy-
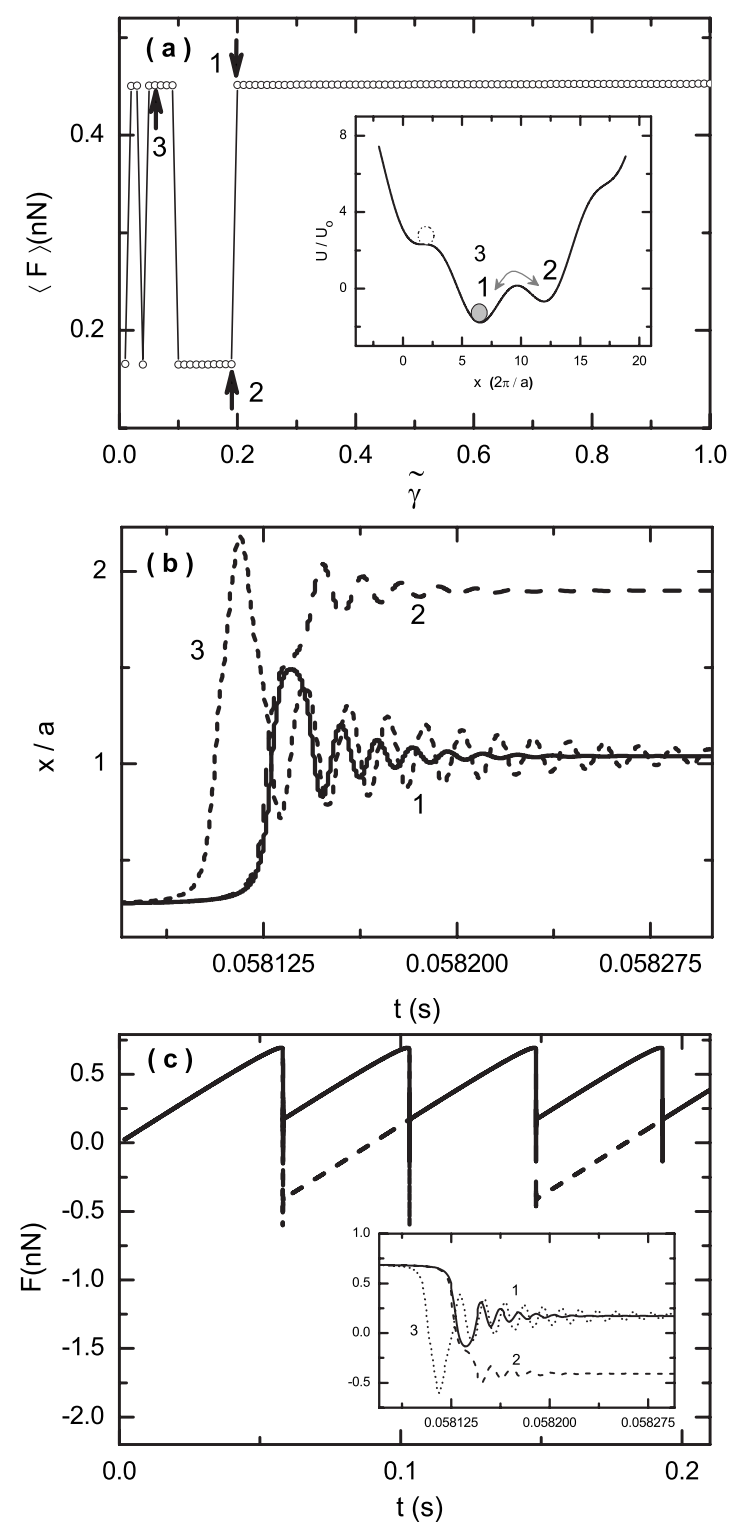

FIG. 7. (a) $\langle F\rangle$ as a function of the dimensionless damping $\tilde{\gamma}$ $\left(\epsilon=0, T=0, U_{0}=0.31\right)$. Time evolution of the tip position (b) and the friction force (c) at $\tilde{\gamma}=0.20$ (curve 1), $\tilde{\gamma}=0.19$ (curve 2), and $\tilde{\gamma}=0.06$ (curve 3 ).

namics results from the interplay between a larger number of minima than that shown in Fig. 7.

When surface disorder and/or temperature are included, it is observed, as expected, that this behavior is strongly smoothed or even suppressed. Figure 8(a) shows the change in the friction force and the mean slip length as a function of the damping for a small disorder $(\epsilon=0.1)$ and its comparison to the regular $(\epsilon=0)$ case curve. Figure $8(\mathrm{~b})$ shows the friction force as a function of the damping when $\epsilon=0.1$ and at three different values of temperature.

\section{DISCUSSION AND CONCLUSIONS}

In order to investigate the effect of surface disorder on atomic friction we have made extensive numerical simula- 

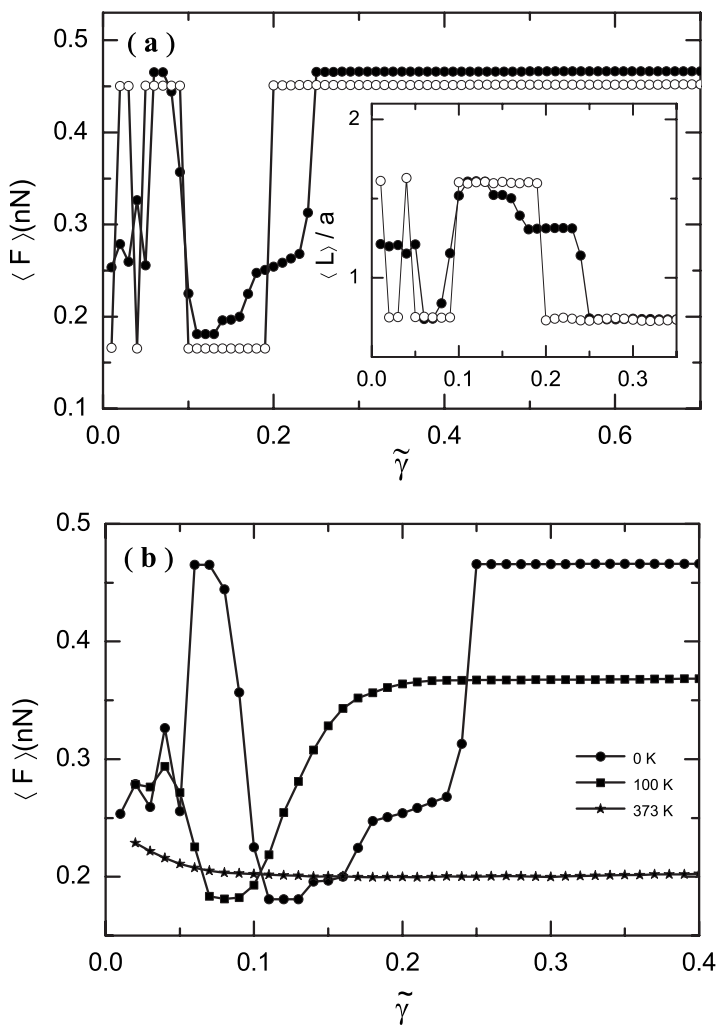

FIG. 8. (a) Effect of surface disorder on the friction force and the mean slip length at different values of the normalized damping parameter: $T=0 \mathrm{~K}$, open symbols stand for $\epsilon=0$ and solid ones for $\epsilon=0.1$. (b) Combined effect of surface disorder $(\epsilon=0.1)$ and temperature $(T=0,100$, and $373 \mathrm{~K})$ on the friction force at different values of the normalized damping. In both cases $U_{0}=0.31 \mathrm{eV}$.

tions based on the one-dimensional Prandtl-Tomlinson model. Our results show the important role played by surface disorder at some given parameter values. In particular, disorder is important at low temperatures. At high-enough temperatures important thermal fluctuations are able to screen the disorder effect. Also surface disorder is particularly important close to dynamical transition points and high values of substrate potential amplitude $U_{o}$. The dynamical transition points are associated to discontinuities in the mean value of the slip length of the stick-slip regimen. Thus, the determination of this mean value has been shown to be very useful in order to understand the dynamical response of the system.

We have approached disorder by including an incommensurate second harmonic in the tip-surface interaction potential. Our model slightly modifies the values of the extreme positions and the barrier heights with respect to the usual regular potential. Although such model does not correspond to a random disorder model, at short length scales differences between both cases are unimportant. In any case, Eq. (2) also represents the potential profile in a given direction for a quasiperiodic lattice. ${ }^{28}$

We have presented results for moderate to intermediate values of the disorder parameter, $\epsilon=0,0.1$, and 0.3 . Obviously, the effect is much stronger at higher values of $\epsilon$. However such potentials represent a strong modification with respect to the regular one. Regarding the commensurability factor $\eta$, we have found similar results for other values of this parameter.

We have focused our attention on the stick-slip region of the force versus velocity curve of the system. Beyond this region the curve is unaffected by disorder or temperature as seen in Figs. 3(b), 4(b), 4(c), and 6. The stick-slip region is dominated by the amplitude of the different stick slips. In the regular case and at zero temperature the dynamics is periodic and the transitions between the different dynamical states, defined by different values of $\langle L\rangle$, are abrupt. Both, disorder and temperature produce a mixing of different length slips. Thus, as shown in the different figures, they smooth out the friction curves in a similar way.

An important issue is the modeling of the tip and its interaction with the environment. This interaction sets the value of the damping of the system. Experimental results have shown that acceptable values of the dimensionless damping are in the overdamped and in the moderate to high damping areas. As we have seen a correct identification of the damping experienced by the tip is important to understand the dynamics of the system.

Regarding the coupling of the tip to the moving support the Prandtl-Tomlinson model approach this interaction in the simplest way, a harmonic coupling. However, recent works which use other coupling schemes show that for some parameter values the dynamical response depends on the model. Tip elasticity has been recently studied for instance using a two-spring-two-masses model. ${ }^{26,29}$ We believe that the effects of disorder in this more complex scheme will be similar to those reported here.

\section{ACKNOWLEDGMENTS}

The authors acknowledge financial support from Spanish MICINN under Project No. FIS2008-01240, cofinanced by FEDER funds. O. Y. Fajardo acknowledges financial support from the Mazda foundation of Colombia, SCH-Unizar, and FPU grant by Ministerio de Ciencia e Inovación of Spain. O. Y. Fajardo wish to thank D. Prada for programming assistance.

\footnotetext{
*yovany@unizar.es

${ }^{1}$ The Friction and Lubrication of Solids, edited by F. P. Bowden and D. Tabor (Oxford University Press, Oxford, 1954).

${ }^{2}$ Fundamentals of Friction: Macroscopic and Microscopic Processes, NATO ASI Series E, edited by I. L. Singer and H. M.
}

Pollock, (Kluwer Academic, Dordrecht, 1992), Vol. 220.

${ }^{3}$ Physics of Sliding Friction, NATO ASI Series E, edited by B. N. J. Persson and E. Tosatti (Kluwer Academic, Dordrecht, 1996), Vol 331.

${ }^{4}$ Fundamentals of Friction and Wear on the Nanoscale, edited by 
E. Gnecco and E. Meyer (Springer, New York, 2007).

${ }^{5}$ M. Urbakh and E. Meyer, Nature Mater. 9, 8 (2010).

${ }^{6} \mathrm{M}$. Urbakh, J. Klafter, D. Gourdon, and J. Israelachvili, Nature (London) 430, 525 (2004).

${ }^{7}$ C. Veigel and C. F. Schmidt, Science 325, 826 (2009).

${ }^{8}$ I. Szlufarska, M. Chandross, and R. W. Carpick, J. Phys. D 41, 123001 (2008).

${ }^{9}$ K. L. Johnson and J. Woodhouse, Tribol. Lett. 5, 155 (1998).

${ }^{10}$ S. N. Medyanik, W. K. Liu, I. H. Sung, and R. W. Carpick, Phys. Rev. Lett. 97, 136106 (2006).

${ }^{11}$ E. Gnecco, R. Bennewitz, T. Gyalog, Ch. Loppacher, M. Bammerlin, E. Meyer, and H.-J. Guntherodt, Phys. Rev. Lett. 84, 1172 (2000).

${ }^{12}$ Y. Sang, M. Dube, and M. Grant, Phys. Rev. Lett. 87, 174301 (2001).

${ }^{13}$ Z. Tshiprut, S. Zelner, and M. Urbakh, Phys. Rev. Lett. 102, 136102 (2009).

${ }^{14}$ Y. Braiman, H. G. E. Hentschel, F. Family, C. Mak, and J. Krim, Phys. Rev. E 59, R4737 (1999).

${ }^{15}$ H. Hölscher, D. Ebeling, and U. D. Schwarz, Phys. Rev. Lett. 101, 246105 (2008).

${ }^{16}$ A. Socoliuc, R. Bennewitz, E. Gnecco, and E. Meyer, Phys. Rev. Lett. 92, 134301 (2004).

${ }^{17}$ Y. Sang, M. Dube, and M. Grant, (Ref. 12) used $a=0.4 \mathrm{~nm}, k$ $=0.93 \mathrm{~N} / \mathrm{m}, \quad U_{o}=0.27 \mathrm{eV}, \quad \gamma=8.9 \times 10^{5} \mathrm{~s}^{-1}, \quad M=8.7$ $\times 10^{-12} \mathrm{~kg}$, and $v_{s}=25 \mathrm{~nm} / \mathrm{s}$ which corresponds to $\tilde{\gamma}=0.804$ and $\Theta=11.48$.
${ }^{18}$ Due to the existence of an early creep stage in the movement of the tip before each slip event, the slip length is not exactly equal to a multiple of the lattice constant.

${ }^{19}$ J. Nakamura, S. Wakunami, and A. Natori, Phys. Rev. B 72, 235415 (2005).

${ }^{20}$ S. Sills and R. M. Overney, Phys. Rev. Lett. 91, 095501 (2003).

${ }^{21}$ E. Riedo and E. Gnecco, Nanotechnology 15, S288 (2004).

${ }^{22}$ A. Schirmeisen, L. Jansen, and H. Fuchs, Phys. Rev. B 71, 245403 (2005).

${ }^{23}$ A. Schirmeisen, L. Jansen, H. Holscher, and H. Fuchs, Appl. Phys. Lett. 88, 123108 (2006).

${ }^{24}$ I. Barel, M. Urbakh, L. Jansen, and A. Schirmeisen, Phys. Rev. Lett. 104, 066104 (2010).

${ }^{25}$ C. Fusco and A. Fasolino, Phys. Rev. B 71, 045413 (2005).

${ }^{26}$ Z. Tshiprut, A. E. Flippov, and M. Urbakh, J. Phys.: Condens. Matter 20, 354002 (2008).

${ }^{27}$ J. Kerssemakers and J. Th. M. Hosson, Appl. Phys. Lett. 67, 347 (1995).

${ }^{28}$ Friction in a quasicrystal has been study in J. Y. Park, D. F. Ogletree, M. Salmeron, R. A. Ribeiro, P. C. Canfield, C. J. Jenks, and P. A. Thiel, Science 309, 1354 (2005); Phys. Rev. B 74, 024203 (2006); A. E. Filippov, A. Vanossi, and M. Urbakh, Phys. Rev. Lett. 104, 074302 (2010).

${ }^{29}$ S. Y. Krylov, J. A. Dijksman, W. A. van Loo, and J. W. M. Frenken, Phys. Rev. Lett. 97, 166103 (2006). 